\title{
Pandemic Equation for Describing and Predicting COVID19 Evolution
}

\author{
Michael Shur ${ }^{1,2}$ (DD
}

Received: 22 May 2020 / Revised: 21 November 2020 / Accepted: 24 November 2020 /

Published online: 7 January 2021

(C) The Author(s), under exclusive licence to Springer Nature Switzerland AG part of Springer Nature 2021

\begin{abstract}
The purpose of this work is to describe the dynamics of the COVID-19 pandemics accounting for the mitigation measures, for the introduction or removal of the quarantine, and for the effect of vaccination when and if introduced. The methods used include the derivation of the Pandemic Equation describing the mitigation measures via the evolution of the growth time constant in the Pandemic Equation resulting in an asymmetric pandemic curve with a steeper rise than a decrease and mitigation measures. The Pandemic Equation predicts how the quarantine removal and business opening lead to a spike in the pandemic curve. The effective vaccination reduces the new daily infections predicted by the Pandemic Equation. The pandemic curves in many localities have similar time dependencies but shifted in time. The Pandemic Equation parameters extracted from the well advanced pandemic curves can be used for predicting the pandemic evolution in the localities, where the pandemics is still in the initial stages. Using the multiple pandemic locations for the parameter extraction allows for the uncertainty quantification in predicting the pandemic evolution using the introduced Pandemic Equation. Compared with other pandemic models our approach allows for easier parameter extraction amenable to using Artificial Intelligence models.
\end{abstract}

Keywords COVID19 · Pandemic · Mitigation · Quarantine

\section{Introduction}

The management of the COVID-s19 pandemics requires a difficult trade-off between limiting the spread of the disease and trying to control and limit the horrendous damage to the economy. Opening the USA could limit the damage to the economy and relieve

Michael Shur

michael.shur@electronicsoffuture.com; shurm@ rpi.edu; https://www.electronicsoffuture.com

1 Rensselaer Polytechnic Institute, Troy, NY, USA

2 Electronics of the Future, Inc., Vienna, VA 22181, USA 
economic and psychological pressures but could also lead to a spike in the COVID-19 infections. To address this problem, many states have chosen a partial opening. For example, most of the state of Virginia was open on May 14, 2020. However, Northern Virginia, Richmond, and Accomack County were all granted 2-week delays.

The predictive models could guide the pandemic response by learning from the past [1]. Some are based on Lotka-Volterra (prey-predator) equation [2], others use comparisons with previous epidemics [3]. There are many attempts to address more specific issues, such as a lockdown impact [4] or explore the evolution of Intensive Unit bed availability [5] or effects of tracing [6]. Some of the models focus on predicting the average number of infections caused by an infected person [7], the effects of the social contact patterns [8] and estimating the severity of the coronavirus disease [9]. The models range from those assessing the global impact of the COVID-19 pandemic [10] to the models focusing on European countries [11] [12], Asia [13] (including China) [14] and on the low- and middle-income countries [15] [16], with the latter addressing opposing effects of the limited health care options and having a younger population on the pandemic trends. The pandemic models also address the effects of the seasonal variations on the pandemic growth [17]. Many of the COVID-19 models are presented at the Center for Disease Control and Prevention COVD-19 forecasting site [18] and ForecastHub [19]. Predictive mathematical models of the COVID-19 Pandemic are reviewed in Reference [20] It has been even claimed that simulations drive the world's response to COVID-19 [21]. Models, such as Advanced Autoregressive Integrated Moving Average (ARIMA) Model [22], have been used to predict the trends of the COVID-19 pandemic for the most affected countries. The factors affecting the accuracy of such models have been widely discussed (see, e.g., [23] and references therein). They include uncertainties in estimating asymptotic infections and time lag between infections and deaths, possible underestimation of the COVID-19 deaths [24], [25], issues related to establishing COVID-19 recovery [26], and strikingly different patterns of the pandemic in different countries and even in the different regions of the same country [27] [28]. The challenging problem is to account for the balance between controlling the pandemic growth and negatively affecting the economy [29] [ 30]. , These difficulties and uncertainties highlight the case for the development of the unified model with an established parameter extraction procedure and parameters that have a clearly understood meaning and could be related to the factors, such as mitigation measures or factors specific to a given locality.

I now propose a unified model based on a new equation that I call the Pandemic Equation. This equation has as simple analytical form but could accurately fit different pandemic evolution curves and account for the effects of mitigation measures and/or of opening and closing the economy. This allows for establishing a balance between limiting the spread of the virus and the economic hardship. The equation could be used to predict pandemic trends. The predictive time range of the Pandemic Equation could be estimated by using a partial set of the fitted pandemic data for the prediction of the pandemic evolution. The calculated results could be then compared with the entire available data set. The results of such a procedure could be used for the uncertainty quantification of the future pandemic evolution. The parameter extraction and the uncertainty quantification procedures could be incorporated into trainable Artificial Intelligence models for the big data processing approach to the pandemics. 


\section{Pandemic Equation}

The unique feature of our model is having a limited number of the pandemic curve evolution parameters with an easily understood meaning and well-defined parameter extraction procedure. The model parameters are directly linked to the mitigation measures, such as the introduction or removal of the quarantine, and to the effect of vaccination when and if introduced. The quarantine removal and business opening lead to a spike in the pandemic curve predicted by Pandemic equation. The effective vaccination reduces the new daily infections described by the Pandemic equation. The pandemic curves in many localities have similar time dependencies but shifted in time. The Pandemic Equation parameters extracted from the well-advanced pandemic curves can be also used for predicting the pandemic evolution in the localities, where the pandemics is still in the initial stages or having a rebound. Using the multiple pandemic locations for the parameter extraction allows for the uncertainty quantification in predicting the pandemic evolution. Future work will use artificial intelligence to train the Pandemic Equation model for the parameter optimization based on the history of multiple pandemic locations.

The Pandemic Equation could be applied to other pandemics using different parameter sets. It could be also find applications for describing other processes, such as stock price evolution that could be correlated with the market conditions and management changes.

Table 1 summarizes the parameters of the Pandemic Equation curve and relates these parameters to specific events and measures during the pandemic.

Table 1 Pandemic Equation curve parameters and related characteristics

\begin{tabular}{|c|c|c|c|}
\hline Parameter & Unit & Meaning & Comment \\
\hline$N$ & & $\begin{array}{l}\text { Number of people infected from } \\
\text { pandemic start }\end{array}$ & \\
\hline$N_{t}$ & - & $\begin{array}{l}\text { Total number of people who } \\
\text { could be infected }\end{array}$ & . \\
\hline$N_{o}$ & & $\begin{array}{l}\text { Number of infected people } \\
\text { at pandemic start }\end{array}$ & Typical values 1 to 20 \\
\hline$f_{o}$ & - & Initial infection ratio & $f_{o}=N_{o} / N_{t}$ \\
\hline$\tau_{o}$ & day & Initial growth time constant & Typical values from 2 to 5 days \\
\hline$\tau$ & day & Time dependent growth time constant & $\tau=\tau_{o}+\alpha t$ \\
\hline$\alpha$ & - & Curve flattening parameter $\left(\tau=\tau_{o}+\alpha t\right)$ & $\begin{array}{l}\alpha \text { is extracted from } \\
\text { pandemic peak time, } t_{m}\end{array}$ \\
\hline$\beta_{1}, \beta_{2} \ldots \beta_{\mathrm{n}}$ & - & $\begin{array}{l}\text { Mitigation parameters for } \\
\qquad 1,2, \ldots n \text { Mitigation events }\end{array}$ & $\begin{array}{l}\text { Negative } \beta \text { correspond to } \\
\text { lifting restrictions. Typical } \\
\text { values }-3 \text { to } 1\end{array}$ \\
\hline$t_{\beta 1}, t_{\beta 2 \ldots} t_{\beta n}$ & day & Times of mitigation events & $\begin{array}{l}\text { Typically larger that the } \\
\text { pandemic peak time }\end{array}$ \\
\hline$\tau_{\beta 1}, \tau_{\beta 2} \ldots \ldots \tau_{\beta n}$ & day & Time constants of mitigation events. & $\begin{array}{l}\text { Typically very small } \\
\text { From } 1 / N_{t} \text { to } 10 / N_{t}\end{array}$ \\
\hline$t_{m}$ & day & Time of the pandemic peak & $\begin{array}{l}\text { Time of the first peak for } \\
\text { multiple peaks }\end{array}$ \\
\hline
\end{tabular}


As seen from Table 1, all parameters of the Pandemic Equation have a very clear meaning and, as shown below, their y extraction from the pandemic evolution data is straightforward.

We start from the standard rate equation (see, for example [31, 32],) that we generalize to obtain the Pandemic Equation.

$$
\frac{d N}{d t}=\frac{\left(1-N / N_{t}\right) N}{\tau_{o}}
$$

Here $N$ is the total number of infections (not of the infected people because some infected people recovered and some, unfortunately died), $\tau_{o}$ is the characteristic time of the pandemic growth, and $N_{t}$ is the total number of people who could be infected (the infection pool). $N_{t}$ is smaller than the number of people $N_{p}$ in the population because some people could have genetic immunity to the virus or isolated from the community. The herd immunity ratio

$$
f_{\text {herd }}=N_{t} / N_{p}<1
$$

was estimated to be $60-70 \%$ [33], but it could strongly depend on a specific locality, since it depends on the transmission ratio that varies a great deal depending on the population's habit (e.g., it could be much smaller in South Korea, where people have been used to wearing masks than in the USA).

From Eq. (1), the ratio of the total infection to the infection pool $f=N / N_{t}$ is

$$
\frac{d f}{d t}=\frac{(1-f) f}{\tau_{o}}
$$

The solution of Eq. (3) is

$$
f=\frac{\exp \left(t / \tau_{o}\right)}{\exp \left(t / \tau_{o}\right)+\exp \left(C_{1}\right)}
$$

Here $C_{l}$ is the integration constant to be determined form the initial conditions $f(0)=f_{o}$

$$
f_{o}=\left(1+e^{C_{1}}\right)^{-1}
$$

Since $f_{o}<<1, f_{o} \approx e^{-C_{1}}$ and Eq. (4) becomes

$$
f=\frac{f_{o} \exp \left(t / \tau_{o}\right)}{f_{o} \exp \left(t / \tau_{o}\right)+1}=\frac{f_{o} \exp (x)}{f_{o} \exp (x)+1}
$$


Hence, the total number of the infected people $N_{i o}$ is given by

$$
N=\frac{N_{t} f_{o} \exp (x)}{f_{o} \exp (x)+1}
$$

Here $x=t / \tau$. For following the pandemic evolution, the daily number of the new infections $\Delta N$ is more important than the total number of infections to date. From Eq. (7)

$$
\Delta N=\frac{d N_{t o}}{d t}=\frac{N_{t o} f_{o} e^{t / \tau_{o}}}{\left(1+e^{t / \tau_{o}} f_{o}\right)^{2} \tau_{o}}
$$

As seen from Fig. 1, this approach to modeling pandemic might only work at the initial stage of the pandemics (during its exponential growth).

The maximum of this symmetric curve is reached at

$$
t_{m o}=\tau \ln \left(1 / f_{o}\right)
$$

The pandemic evolution should be described by a more complex time dependence of the new infections with a typical asymmetrical curve. This could be accounted for by making the pandemic growth time constant 7 and the infection pool $N_{t}$ to be a slow (on the scale of $\tau_{o}$ ) functions of time:

$$
\Delta N_{i}=\frac{N_{t}(t) f_{o} e^{t / \tau(t)}}{\left(1+f_{o} e^{t / \tau(t)}\right)^{2} \tau(t)}
$$

This approach is similar to the adiabatic approximation in the quantum theory of solids of allowing two time scales for slow (atomic) and rapid (electronic) motion [34]. We call Eq. (10) the Pandemic Equation.

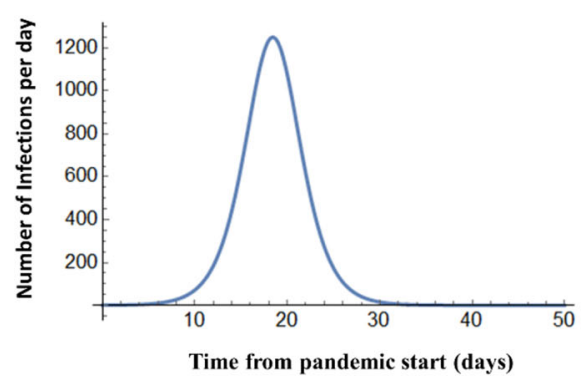

(a)

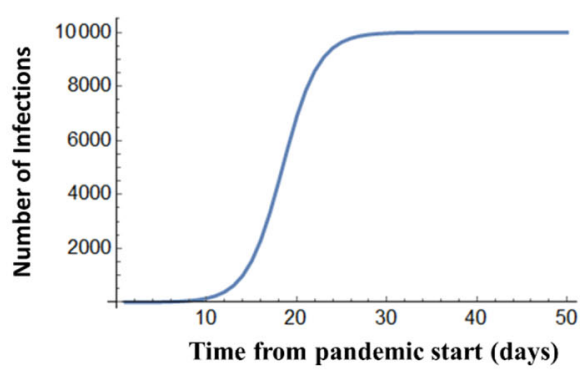

(b)

Fig. 1 Solutions of the rate equation: the number of infected people and the number of people infected per day as a function of time from pandemic start 
The total number of the infected people as a function of time is then given by

$$
N_{i}(t)=\int_{0}^{t} \Delta N_{t}\left(t^{\prime}\right) d t^{\prime}
$$

We will show that we could fit a variety of data using the following function

$$
\tau=\tau_{o}+\alpha t
$$

leading to

$$
\Delta N_{i}=\frac{N_{t \alpha}(\alpha) f_{\alpha} e^{t /\left(\tau_{o}+\alpha t\right)}}{\left(1+f_{\alpha} e^{t /\left(\tau_{o}+\alpha t\right)}\right)^{2}\left(\tau_{o}+\alpha t\right)}
$$

Here $\alpha$ is the flattening parameter responsible for "flattening the curve" as illustrated by Fig. 2(a) showing an asymmetric pandemic curves reproduced using the time dependence of $\tau$.

The maxima of the daily infections reached at approximately

$$
t_{m} \approx \frac{\tau_{o} \ln \left(1 / f_{o}\right)}{1-\alpha \ln \left(1 / f_{o}\right)}
$$

This expression is obtained from Eq. (13) assuming that $\alpha<<\tau_{o} / t_{m}$, so that the terms $\alpha t$ are only kept in the exponential functions in Eq. (13). Figure 2(b) shows the dependence given by Eq. (14). As seen, Eq. (14) agrees well with the position of the maxima in Fig. 2(a) and it could be used for the parameter extraction. Our numerical calculations show that Eq. (14) works as a reasonable approximation if $\alpha<<t_{m o}=$ $\ln \left(1 / f_{o}\right)$. As seen from Fig. 2(a), varying the curve flattening parameter a reproduces well different degrees of the curve flattening and, as seen from Fig. 2(b) could be easily

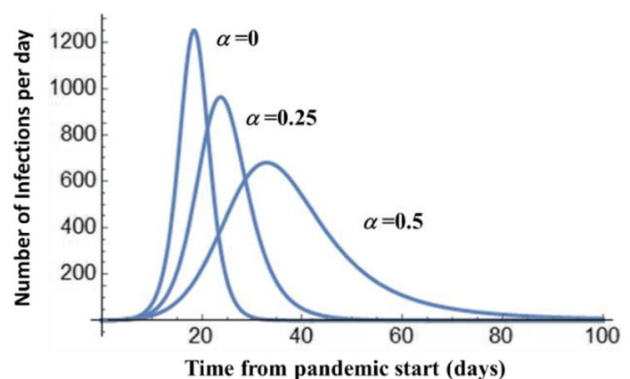

(a)

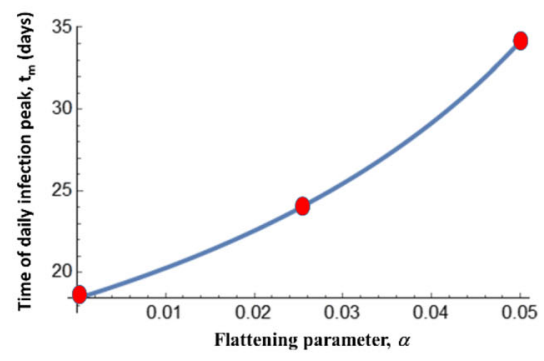

(b)

Fig. 2 a Number of people infected per day as a function of time from pandemic start. b Time of the pandemic peak versus flattening parameter (solid line calculated using Eq. (14), dots are calculated numerically) (b). Parameters used in the calculation: $N_{t}=N_{t \alpha}=10,000, f_{\alpha}=1 / N_{t a}, \tau_{0}=2$ days 
extracted based on the initial time constant of the pandemic growth and the time of the first pandemic peak.

We now introduce the effect of the mitigation measures, such as contact tracing, closing or opening the economy, introduction or removal or the quarantine, and requiring wearing masks and social distancing in public places. Now the Pandemic Equation becomes

$$
\Delta N_{\beta}(t)=\Delta N_{i}(t, \alpha)\left(1-\beta /\left(1+\exp \left(\frac{t_{\beta}-t}{\tau_{\beta}}\right)\right)\right)
$$

Here $\beta$ is the mitigation parameter, $\tau_{\beta}$ is the characteristic time constant of the control measures introduction, and $t_{\beta}$ is the characteristic time of the control measures introduction (the effect of the measures starts at approximately $t_{\beta}-3 \tau_{\beta}$ and finishes at approximately $\left.t_{\beta}+3 \tau_{\beta}\right)$.

The Pandemic Equation could be further generalize to account for multiple government mitigation events as follows

$$
\begin{aligned}
& \Delta N_{\beta_{1}, \beta_{2} \cdots \beta_{n}}(t)=\Delta N_{i}(t, \alpha)\left(1-\frac{\beta_{1}}{1+\exp \left(\left(t-t_{\beta 1}\right) / \tau_{\beta 1}\right)}\right) \times \\
& \left(1-\frac{\beta_{2}}{1+\exp \left(\left(t-t_{\beta 2}\right) / \tau_{\beta 2}\right)}\right) \cdots\left(1-\frac{\beta_{n}}{1+\exp \left(\left(t-t_{\beta n}\right) / \tau_{\beta n}\right)}\right)
\end{aligned}
$$

\section{Results and Discussion}

We start from simulating different mitigation effects using the Pandemic Equation and then discuss the parameter extraction that we apply to the COVID19 data for New City and the Commonwealth of Virginia.

Figure 3 shows the simulated effect of vaccination for different vaccine efficiencies represented by the values of the mitigation parameter $\beta=0.1,0.5$, and 1 . The value of $\beta=1$ corresponds to the limiting case of the totally effective vaccination.) The ability of our model to account for the vaccination effectiveness is very important. Typically, for
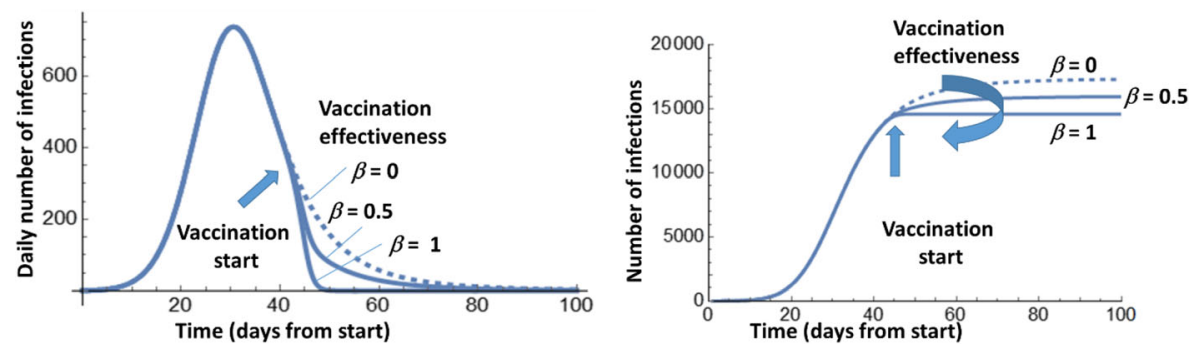

Fig. 3 Simulated effect of vaccination for different vaccine efficiencies represented by $\beta=0.1,0.5$, and 1 
a flu vaccination, the vaccine effectiveness is between 40 and $60 \%$ according to CDC [35]. Recent reports estimate the COVID-19 vaccine effectiveness to be approximately $90 \%$. However, the US public, for example, is divided over whether to get the COVID19 vaccine [36]. All these factors could be accounted for by the values of the mitigation parameter corresponding to vaccination and could be reproduced by varying the mitigation parameter. It is also important that the model accounts for the characteristic time of the vaccine deployment.

Figure 4 shows the simulated effects of relaxing the COVID-19-induced restrictions and opening the economy. We could relate the values of $\beta=-1$ and $\beta=-2$ to Phase 1 and Phase 2 of lifting the COVID-19-related restrictions.

Figure 5 shows relaxing the simulated combined effect of lifting and re-introducing restrictions, such as opening and the closing down economy. As seen, the Pandemic Equation reproduces well the complicated shapes of the pandemic evolution by accounting for multiple events corresponding to positive and negative values of the mitigation parameter and related time constants.

We now use the Pandemic Equation for fitting the pandemic evolution curves for the Commonwealth of Virginia and New York City. The parameter extraction for the Pandemic Equation starts from plotting the number of the natural log of the total infections as a function of time. This dependence is linear at the beginning of the pandemic. The slope and intercept of this line yield $\tau_{o}$ and $f_{o}$, respectfully. From the time of the first peak, we estimate parameter $\alpha$ using Eq. (17)

$$
\alpha \approx \frac{t_{m}-\tau_{o} \ln \left(1 / f_{o}\right)}{t_{m} \ln \left(1 / f_{o}\right)}
$$

Parameter $N_{t}$ is then extracted from fitting the peak value of the daily infections. Finally, we choose the values of parameters $\beta, t_{\beta}$ and $\tau_{\beta}$. Figure 6 and Fig. 7 show the resulting fits.

The Pandemic Equation is capable of reproducing the pandemic evolution curves with multiple events leading to the second spike. However, a more important application of the Pandemic Equation in its predictive capability to be endowed by the parameter extraction and interpretation from the localities, where the pandemic has run its course or at least the pandemic is far enough on the decreasing slope. Future work should include developing the AI approach to train the Pandemic Equation model
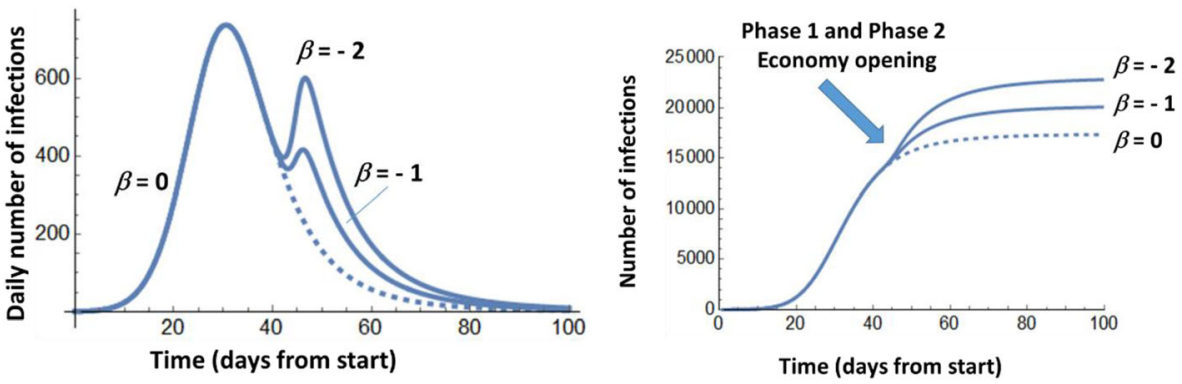

Fig. 4 Simulated effects of relaxing the COVID19 induced restrictions and opening the economy 


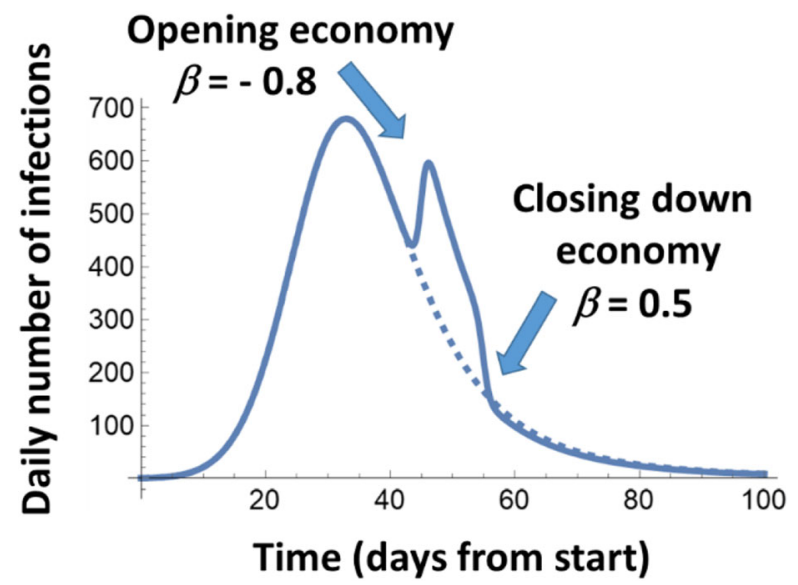

Fig. 5 Combined effect of lifting and re-introducing restrictions, such as opening and the closing down economy

for the parameter optimization based on the history of multiple pandemic locations with well advanced pandemic history.

Fitting the data for New York City and Commonwealth of Virginia pandemic evolution revealed interesting trends. The fitted values of the population pools for the infection ( $10^{5}$ for NYC and $2.4 \times 10^{4}$ for Commonwealth of Virginia) are much smaller than the total New York and Virginia populations, respectively. Also, the curve flattening parameters are quite large ( 0.067 for Virginia and 0.0631 for NYC). We speculate that these results could be explained by having loosely connected infection

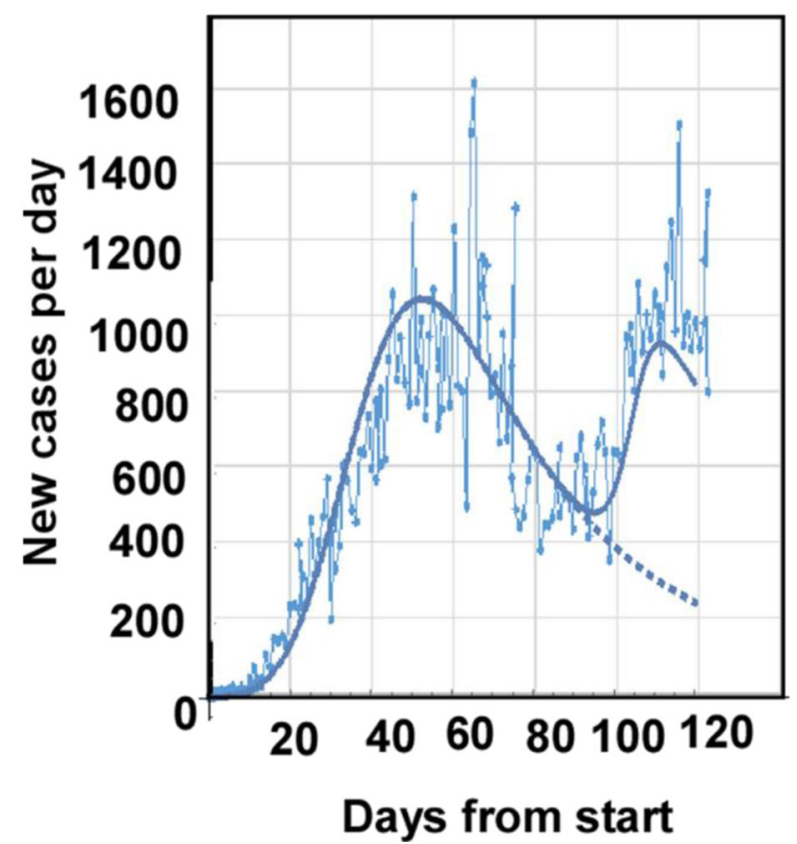

Fig. 6 Fitting the Commonwealth of Virginia pandemic evolution (solid line: the Pandemic Equation fit) 


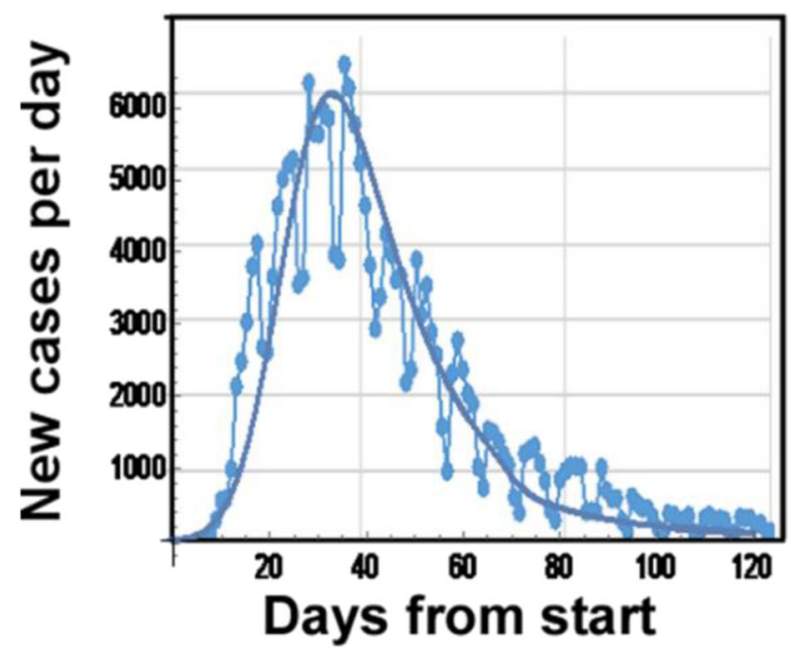

Fig. 7 Fitting the NYC pandemic evolution (solid line: the Pandemic Equation fit)

clusters. One such cluster could be international fliers who brought the initial infection from China or Italy and their friends and relatives belonging to the NYC more affluent society. Another cluster might be low-income population that includes waiters, porters, and cab drivers getting infected serving the original infected people. Still another cluster in NYC is the nursing home population. A large fraction of the population has remained relatively unaffected. It is also possible that more affected individuals transmit the decease at a higher rate but they die or recover first making the transmission less likely. The NYC data also reveal a very long low infection rate tail. The duration of this tail allows to estimate the low limit of the acquired immunity, which seems to exceed four months. More fitting by the Pandemic Equation for different localities and longer periods of time might prove or disprove these speculations but they show that presenting the noise pandemic data by a few clear parameters driving the pandemic is very useful for the analysis of the trends and predicting our future.

The evolution of the new cases for Virginia (see Fig. 6) is very different. If we again apply the cluster hypothesis to the pandemic evolution a much smaller per capita first peak for the Virginia pandemic means that the intra-cluster herd immunity has not been reached in contrast to the New York situation and the rebound potential was higher when the state was opening up.

\section{Conclusion}

The Pandemic Equation is the modified rate equation with slowly varying parameters. This approach is similar to the adiabatic approximation in quantum theory of solids. The Pandemic Equation describes the pandemic evolution curves accounting for curve flattening and mitigation measures, such as the introduction or removal of the quarantine and for the effect of vaccination when and if introduced. Extracting these parameters from the well-advanced pandemic curves allows for reaching conclusions about the pandemic trends and might enables the Pandemic Equation to predict the pandemic 
evolution for the locations where the pandemics is not yet very advanced including the effects of different mitigation measures.

Future work should include linking the model parameters to different measures and events affecting pandemic. The simplicity and relative ease of the parameter extraction should make this model to be a tool that could be widely used by local governments and communities for understand the past and trying to predict the future trends. This work could be supplemented by an artificial intelligence model to be trained to recognize the features of the pandemic curves and automatically extract the model parameter, similar to how it was done for extracting the image features during integrated circuit testing [37].

Funding No funding sources have been used.

Data Availability Not applicable.

\section{Compliance with Ethical Standards}

Conflicts of Interest/Competing Interests The author has not conflicting interests and no competing interests.

Code Availability custom code.

\section{References}

1. J. E. Metcalf, D. H. Morris, and S. W. Park, Mathematical models to guide pandemic response, Science, Issue 6502, 24 July, p. 369 (2020)

2. Berryman AA (1992) The origins and evolution of predator-prey theory. Ecology. 73(5):1530-1535

3. Patrice Bourdelais, Mapping the course of an epidemic: the example of two cholera epidemics in France (1832 and 1854), http://cams.ehess.fr/modeling-the-propagation-of-covid-19-abstracts/\#bourdelais

4. L. Di Domenico and V. Colizza (EPIcx lab, INSERM, Sorbonne Université), Expected impact of lockdown on COVID-19 epidemic in Île-de-France and possible exit strategies, http://cams.ehess.fr/ modeling-the-propagation-of-covid-19-abstracts/\#colizza

5. G. Dulac-Arnold and J.-P. Nadal (Intensive Care Unit Bed Availability Monitoring and Modelling during the COVID-19 Epidemic in the Grand Est region of France, http://cams.ehess.fr/modeling-thepropagation-of-covid-19-abstracts/\#ferretti

6. L. Zdeborová (CEA Saclay), F. Krzakala and M. Mézard (ENS Paris), Alfredo Braunstein (Politecnico di Torino) et al, Risk estimation from contact tracing data, http://cams.ehess.fr/modeling-the-propagationof-covid-19-abstracts/\#zdeborova

7. Russell TW, Hellewell J, Jarvis CI, van Zandvoort K, Abbott S, Ratnayake R, CMMID COVID-19 working group, Flasche S, Eggo RM, Edmunds WJ, Kucharski AJ (2020) Estimating the infection and case fatality ratio for coronavirus disease (COVID-19) using age-adjusted data from the outbreak on the diamond princess cruise ship. Euro Surveill 25:2000256. https://doi.org/10.2807/1560-7917.ES.2020.25. 12.2000256

8. Leung K, Jit M, Lau EHY, Wu JT (2017) Social contact patterns relevant to the spread of respiratory infectious diseases in Hong Kong. Sci Rep 7:7974. https://doi.org/10.1038/s41598-017-08241-1pmid: 28801623

9. R. Verity, L. C. Okell, I. Dorigatti, P. Winskill, C. Whittaker, N. Imai, G. Cuomo-Dannenburg, H. Thompson, P. G. T. Walker, H. Fu, A. Dighe, J. T. Griffin, M. Baguelin, S. Bhatia, A. Boonyasiri, A. Cori, Z. Cucunubá, R. FitzJohn, K. Gaythorpe, W. Green, A. Hamlet, W. Hinsley, D. Laydon, G. Nedjati-Gilani, S. Riley, S. van Elsland, E. Volz, H. Wang, Y. Wang, X. Xi, C. A. Donnelly, A. C. Ghani, N. M. Ferguson, Estimates of the severity of coronavirus disease 2019: a model-based analysis. 
Lancet Infect Dis 20, 669-677 (2020). doi:https://doi.org/10.1016/S1473-3099(20)30243-7pmid: $32240634,669,677$

10. Cobey S (2020) Modeling infectious disease dynamics, science, 368. Issue 6492:713-714

11. S. Flaxman, S. Mishra, A. Gandy, J. T. Unwin, H. Coupland, T. A. Mellan, et al., Estimating the number of infections and the impact of non-pharmaceutical interventions on COVID-19 in 11 European countries (Imperial College COVID-19 Response Team) (2020); 10.25561/77731.doi:10.25561/77731

12. H. Salje, C. Tran Kiem, N. Lefrancq, N. Courtejoie, P. Bosetti, J. Paireau, A. Andronico, N. Hozé, J. Richet, C.-L. Dubost, Y. Le Strat, J. Lessler, D. Levy-Bruhl, A. Fontanet, L. Opatowski, P.-Y. Boelle, S. Cauchemez, Estimating the burden of SARS-CoV-2 in France. Science eabc3517 (2020). doi:https://doi. org/10.1126/science.abc3517pmid:32404476

13. .J. Phua, M. O. Faruq, A. P. Kulkarni, I. S. Redjeki, K. Detleuxay, N. Mendsaikhan, K. K. Sann, B. R. Shrestha, M. Hashmi, J. E. M. Palo, R. Haniffa, C. Wang, S. M. R. Hashemian, A. Konkayev, M. B. Mat Nor, B. Patjanasoontorn, K. M. K. Nafees, L. Ling, M. Nishimura, M. J. Al Bahrani, Y. M. Arabi, C.-M. Lim, W.-F. Fang; Asian Analysis of Bed Capacity in Critical Care (ABC) Study Investigators, and the Asian Critical Care Clinical Trials Group, Critical Care Bed Capacity in Asian Countries and Regions. Crit. Care Med. 48, 654-662 (2020). doi:https://doi.org/10.1097/CCM.0000000000004222pmid: 31923030

14. Q. Bi, Y. Wu, S. Mei, C. Ye, X. Zou, Z. Zhang, X. Liu, L. Wei, S. A. Truelove, T. Zhang, W. Gao, C. Cheng, X. Tang, X. Wu, Y. Wu, B. Sun, S. Huang, Y. Sun, J. Zhang, T. Ma, J. Lessler, T. Feng, Epidemiology and transmission of COVID-19 in 391 cases and 1286 of their close contacts in Shenzhen, China: A retrospective cohort study. Lancet Infect. Dis. 0, S1473-3099(20)30287-5 (2020). doi:https:// doi.org/10.1016/S1473-3099(20)30287-5pmid:32353347

15. Walker PGT et al (2020) The impact of COVID-19 and strategies for mitigation and suppression in lowand middle-income countries. Science 369:413

16. Grijalva CG, Goeyvaerts N, Verastegui H, Edwards KM, Gil AI, Lanata CF, Hens N (2015) RESPIRA PERU project. A household-based study of contact networks relevant for the spread of infectious diseases in the highlands of Peru PLOS ONE 10(e0118457). https://doi.org/10.1371/journal.pone.0118457pmid: 25734772

17. Baker RE et al (2020) Susceptible supply limits the role of climate in the early SARS-CoV-2 pandemic. Science 369:315

18. https://www.cdc.gov/coronavirus/2019-ncov/covid-data/forecasting-us.html

19. https://covid19forecasthub.org/doc/participate/

20. Jewell NP, Lewnard JA, Jewell BL (2020) Predictive mathematical models of the COVID-19 pandemic: underlying principles and value of projections. JAMA. 323(19):1893-1894. https://doi.org/10.1001/ jama.2020.6585

21. Adam D (2020) Special report: the simulations driving the world's response to COVID-19. Nature. 580(7803):316-318

22. Singh RK, Rani M, Bhagavathula AS, Sah R, Rodriguez-Morales AJ, Kalita H, Nanda C, Sharma S, Sharma YD, Rabaan AA, Rahmani J, Kumar P (2020) Prediction of the COVID-19 pandemic for the top 15 affected countries: advanced autoregressive integrated moving average (ARIMA) model. JMIR Public Health Surveill 6(2):e19115. https://doi.org/10.2196/19115

23. Petropoulos F, Makridakis S (2020) Forecasting the novel coronavirus COVID-19. PLoS One 15(3): e0231236. https://doi.org/10.1371/journal.pone.0231236

24. Woolf SH, Chapman DA, Sabo RT, Weinberger DM, Hill L (2020) Excess deaths from COVID-19 and other causes, march-April 2020. JAMA. 324(5):510-513. https://doi.org/10.1001/jama.2020.11787

25. Alwan NA, Surveillance is underestimating the burden of the COVID-19, pandemic, www.thelancet.com Vol 396 September 5, 2020

26. Alwan NA (2020) A negative COVID-19 test does not mean recovery. Nature. 584:170

27. Middelburg RA, Rosendaal FR (2020) COVID-19: how to make between-country comparisons. Int J Infect Dis 96:477-481. https://doi.org/10.1016/j.ijid.2020.05.066

28. Bilinski A, Emanuel E (2020) J COVID-19 and excess all-cause mortality in the US and 18 comparison countries. JAMA Published online October 12:2100-2102. https://doi.org/10.1001/jama.2020.20717

29. Haushofer J, Metcalf CJE (2020) Which interventions work best in a pandemic? Science 368:1063-1065

30. P. Klepac et al., Synthesizing epidemiological and economic optima for control of immunizing infections, Proc. Natl. Acad. Sci. U.S.A. 108, 14366

31. Atkins, Peter; de Paula, Julio (2006). "The rates of chemical reactions". Physical chemistry (8th Ed.) W.H. Freeman. pp. 791-823. ISBN 0-7167-8759-8

32. Connors KA (1990) Chemical kinetics: the study of reaction rates in solution. John Wiley \& Sons. isbn: 9781560810063 
33. https://www.mdanderson.org/cancerwise/what-is-covid-19-coronavirus-herd-immunity-when-will-weachieve-herd-immunity.h00-159383523.html

34. M. S. Shur, Physics of Semiconductor Devices (with microcomputer programs), Prentice Hall, New Jersey (1990) ISBN 0-13-666496-2

35. https://www.cdc.gov/flu/vaccines-work/vaccineeffect.htm

36. A. Tyson, C. Johnson and C. Funk U.S. Public Now Divided over Whether to Get COVID-19 Vaccine Concerns about the safety and effectiveness of possible vaccine, pace of approval process. https:// www.pewresearch.org/science/2020/09/17/u-s-public-now-divided-over-whether-to-get-covid-19-vaccine/, September 17, 2020

37. N. Akter, M. Karabiyik, A. Wright, M. Shur and N. Pala, "AI Powered THz Testing Technology for Ensuring Hardware Cybersecurity," (2020) IEEE research and applications of photonics in defense conference (RAPID). Miramar Beach, FL, USA 2020:1-2. https://doi.org/10.1109/RAPID49481.2020. 9195662

Publisher's Note Springer Nature remains neutral with regard to jurisdictional claims in published maps and institutional affiliations. 